meiner Versuche in einem $K_{0}$ chen bestand, so kann von ungenügend hoher Temperatur nicht die Rede sein; auch hatte ich die von M. u. M. vorgeschriebene Zeit, nämlich $z$ wei Stunden lang, gekocht.

Um nun auch den Einwand abzuschneiden, dass man vielleicht bei längerem Kochen doch die salpetrige Săure austreiben könne, habe ich Herrn Zaloziecki veranlasst, einige Versuche in dieser Richtung anzustellen.

Reine concentrirte Schwefelsäure wurde mit so viel Natriumnitrit versetzt, dass dies in 100 cc einer Menge von $0,406 \mathrm{~N}_{2} \mathrm{O}_{3}$ entsprach. Es wurde nun 6 Stunden lang mit eingelegter Platinspirale gekocht, wobei etwa die Hälfte der Säure fortging, der Rückstand mit reiner Säure wieder auf $100 \mathrm{cc}$ gebracht und wieder mit Chamäleon titrirt, wobei nun $0,385 \mathrm{~g} \mathrm{~N}_{2} \mathrm{O}_{3}$ gefunden wurden, entsprechend einem Verluste von 5,2 Proc. des Gesammtgehaltes an $\mathrm{N}_{2} \mathrm{O}_{3}$. Bei einem sweiten Versuche wurde dieselbe nitrose Säure ebenfalls 6 Stunden, aber mit eingelegten Glascapillaren gekocht und wie oben behandelt; im Rückstand war dann noch $0,3706 \mathrm{~g} \mathrm{~N}_{2} \mathrm{O}_{3}$, also 8,7 Proc. Verlust an $\mathrm{N}_{2} \mathrm{O}_{3}$. Berücksichtigt man nun, dass dieser Verlust grossentheils durch rein mechanisches Herausspritzen von Säure entstand (wesbalb es auch beim zweiten Versuche stärker als beim ersten war, weil die Glascapillaren das Stossen beim Kochen nicht so gut wie die Platinspiralen verhinderten), so findet man, dass selbst bei sechsstündigem, vollstem Kochen (Meldola und Moritz schreiben nur 2 Stunden vor) von einer „Austreibung der salpetrigen Säure" überbaupt gar nicht die Rede ist, dass sich vielmehr die Nitrosylschwefeläure beim längeren Kochen der Säure nur immer mehr concentrirt! Die Vorschrift von Meldola und Moritz zur Reinigung der Schwefelsäure für die Kjeldahl'sche Methode ist also nach wie vor als vollkommen falsch hinzustellen; sie kann nur durch einen merkwürdigen Zufall unschädlich sein, wenn nämlich die zugesetzte Menge Natriumnitrit der in der Säure vorbandenen Menge ron Ammoniaksalz genall entspricht; ein Überschuss von 8alpetriger Säure ist nicht durch beliebiges langes Kochen zu entfernen, sondern wird sich dadurch nur immer mehr concentriren, und die Chemiker müssen also vor Befolgung jener angeblichen Reinigungsvorschrift dringend genannt werden.

\section{Ein neues Verfahren zur Darstellung von Sauerstoff.}

Von

\section{Dr. G. Kassner in Broslau.}

Nachdem ich früher gezeigt hatte (Chemzg. 1889 No. 79 und 81), dass das Ferricyankalium die Eigenschaft besitzt, bei Gegenwart von freiem oder kohlensaurem Alkali eine Lösung von Wasserstoffsuperoxyd unter Entwicklung von Sauerstoff zu zerlegen, lag es nahe, das Verhalten jenes Salzes auch zu der Stammsubstanz des Wasserstoffsuperoxydes zu untersucben.

$\mathrm{Zu}$ diesem $\mathrm{Zwecke}$ brachte ich zunāchst pulverförmiges, etwa 80 proc. Baryumsuperoxyd mit einigen Krystallen rothen Blutlaugensalzes und etwas Wasser zusammen. Ich war nun nicht wenig erstaunt, als diese Mischung obne weitere Erwärmung und ohne Zusatz von Alkali stark aufzubrausen begann und ein Gas entwickelte, welches sich ebenfalls als reiner $\mathrm{S}$ a uerstoff erwies. In dem Grade, als sich das Baryumsuperoxyd zersetzte, verschwand hierbei die goldgelbe Farbe des mittlerweile zur Lösung gekommenen Ferricyankaliums, bis die Mischung endlich fast farblos wurde. Hatte ich nun viel Wasser genommen, so trat röllige Lösung ein und nur die geringen Unreinigkeiten des Baryumsuperoxyds blieben auf dem Boden des Gefässes zurück oder auch ein Überschuss des letzteren, falls nicht genau die äquiralente Menge zugesetzt worden war. Anfangs glaubte ich, dass die Zersetzung $z$ wischen rotbem Blutlaugensalz und Baryumsuperoxyd nur bei Gegenwart von viel Wasser quantitativ verläuft und dass die ganze Menge des entstandenen Nebenproductes in Lösung gehen müsse. Dies ist aber, wie ich alsbald fand, durchaus nicht der Fall. Es entwickelt sich also auch mit wenig Wasser die ganze Menge des disponiblen Sauerstoffs.

Wie ich schon jetzt hervorheben will, ist dies ein Umstand von grosser Wichtigkeit, welcher für die Verwendbarkeit dieser neueu Reaction zur Darstellung von Sauerstoff die weiteste Aussicht eröffnet.

Dass bei dem Zusammenbringen von Baryumsuperoxyd mit Ferricyankalium die Farbe des letzteren unter Entwicklung von Sauerstoffgas völlig verschwindet, deutet darauf bin, dass das Ferricyansalz zu Ferrocyansalz reducirt wird. Indessen besitzt das Ferricyankalium keine Spur von Sauerstoff und seine Formel unterscheidet sich ron der ihm im Eisen- und Cyangehalt äqui- 
valenten des Ferrocyankaliums lediglich durch ein Minus von 2 At. Kalium.

Wenn man nach meinem früheren Vorschlage Sauerstoff aus Wasserstoffsuperoxyd und Ferricyankalium bei Gegenwart von $\mathrm{Ka}$ liumhydrat entwickelt, so kann der chemische Process nur so verlaufen, dass das Molecül des Ätzkalis unter Abspaltung von Sauerstoff, Wasser und Metall zersetzt wird und letzteres in die Formel des Ferricyankaliums unter Rückbildung desselben zu Ferrocyankalium eintritt. In dem vorliegenden Falle ist aber die Gegenwart von freiem Ätzkali ganz ausgeschlossen. Nichtsdestoweniger tritt eine Enttfärbung des Ferricyankaliums, d. h. eine Bildung von Ferrocyansalz ein, was übrigena auch durch die bekannte Reaction auf Eisenoxydsalz nachgewiesen werden kann. Gleichzeitig beobachtet man eine völlige Lösung des Baryumsuperoxyds.

Aus beiden Erscheinungen lässt sich daher folgern, duss die Reaction zwischen Ferricyankalium und Baryumsuperoxyd nur in der Weise stattfinden kann, dass der Sauerstoff nur aus den Baryumsuperoxyd stammt und dass das hierdurch freigewordene A tom Baryun in die Formel des Ferricyankaliums eintritt, um dadurch die Bildung eines Doppelsalzes ron Ferrocyankaliumbaryum zu veranlaseen. Dieses würde die Formel $\mathrm{Fe}(\mathrm{CN})_{2} \mathrm{~K}_{3} \frac{\mathrm{Ba}}{2}(\mathrm{CN})_{4}$ oder besser $\left[\mathrm{Fe}(\mathrm{CN})_{6} \mathrm{~K}_{3}\right]_{2} \mathrm{Ba}$ haben müssen, und der Process rerliefe nach der Gleichung:

$\mathrm{BaO}_{2}+\mathrm{Fe}_{2}(\mathrm{CN})_{6}(\mathrm{KCN})_{5}=\left[\mathrm{Fe}(\mathrm{CN})_{2} \mathrm{~K}_{3}(\mathrm{CN})_{4}\right]_{2} \mathrm{Ba}+\mathrm{O}_{2}$ Thatsächlich entsteht bei dem Zusammenbringen von Baryumsuperoxyd und Ferricyankalium mit wenig Wasser ein deutlich krystallinisches Pulver, welches unter dem Mikruskup hübsch ausgebildete Krystalle zeigt, die doppeltbrechend sind. Löst man den Niederschlag durch Kochen mit viel Wasser auf, so krystallisirt die Verbindung in grösseren, fast farblosen Krystallen, deren reine Lösung neutral reagirt und mit verdünnter Scbwefelsäure, sowie deu Sulfaten. Jarbonaten, Phosphaten der Alkalien farblose Niederschläge gibt, sich also wie die Lösung eines Barytsalzes verhält. Aus der Mutterlauge krystallisirt indessen ein viel gelberes Salz, welches nach qualitativer Reaction weniger Baryum zu enthalten scheint; doch ist auch letzteres noch in beträchtlicher Menge darin enthalten. Eine zu vorläufger Orientirung ausgefíhrte Bestimmung des zuerst krystallisirenden, heller gefärbten Salzes ergab 10,8 Proc. Krystallwasser und 49,6 Proc. Ferrocyan $\mathrm{Fe}(\mathrm{CN})_{8}$, während sich für die Formel Fe (CN), $\left[\mathrm{Ba}(\mathrm{CN})_{2}\right]_{2}$ 54,53 Proc. Ferrocyan berechnet. Das Salz kann daher nahezu als Ferrocyanbaryt betrachtet werden. Es scheint daher, als wenn durch Kochen des Reactionsproductes mit viel Wasser und allmählicher Krystallisation eine Bildung und Trennung von reinem Ferrocyanbaryum und Ferrocyankalium erreicht werden kann.

Dies ist aber nur bei Gegenwart einer reichlichen Menge Wasser möglich. In unserem Falle ist es viel wichtiger, dass bei Anwendung von nur wenig Wasser nahezu die ganze Menge von Ferrocyan, Baryum und Kalium in Gestalt einer schwer löslichen Doppelverbindung verbleibt. Dass dies ein wesentlicher Umstand ist, leuchtet sofort ein, wenn man erwägt, dass dadurch ein Mittel gegeben ist, den gesammten Materialwerth der den Sauerstoff liefernden Stoffe zu erbalten, obne dass man erst nothwendig hat, eine Concentration des Reactionsproductes durch Eindampfen o. dgl. vorzunehmen. Die von mir vorgeschlagene Combination beider Körper, des Baryumsuperoxyds und Ferricyankaliums, bietet daher die Möglichkeit, ohne Umstände und ohne nennenswerthe Apparate überall, wo es sein mag, Sauerstoff im Kleinen wie im Grossen entwickeln zu könnep.

In L,aboratorien und Hörsälen würde ein einfacner Kolben genügen, auf dessen Boden mau die trockene Mischung beider Stoffe schüttet, welche nach Eingiessen ron nur wenig Wasser sofort und in reichlicher Menge Sauerstoff entwickelt.

Verbrennt man in dem Sauerstoff einen Holzspahn oder Schwefel, so ist bei dieser Art der Darstellung auch noch insofern ein Vortheil, als das Reactionsgemisch infolge einer stets vorhandenen Beimengung von Barythydrat alkalisch reagirt und noch reichlich Koblensäure und Schwefligsäure zu binden, den Sauerstoff also in dem über der Flüssigkeit befindlichen Raume möglichst lauge rein und wirksam za erbalten vermag.

Will man das $G$ as in einem Gasometer auffangen, so nimmt man einen nicht $z u$ grossen Kolben, füllt denselben etwa halbvoll mit der Mischung und giesst bis zu $3 /$, seines Inhaltes Wasser hinzu. Der Kolben wird jetzt rasch mit einem gut eingepassten, durchbohrten Kork verschlossen und dann mit einem Gasometer in Verbindung gesetzt.

Die Entwicklung des Gases geht sebr rasch vor sicb; will mau sie laugsamer verlaufen lassen, so empfiehlt es sich, die beiden auf einander reagirenden Stoffe nicht zusammen gemischt, sondern getreuut anzuwenden und allmählich mit einander in Berührung zu bringen.

Man erhālt aus $6,58 \mathrm{~g}$ Ferricyankalium und $2,25 \mathrm{~g} 75$ proc. Baryumsuperoxyd wach 
der Theorie $0,32 \mathrm{~g}$ Sauerstoff; ich bekam $240 \mathrm{cc}$ von $15^{\circ}$, was mit der Rechnung ziemlich stimmt, nach welcher (unter Vernachlässigung des Barometers und der Tension der Wasserdämpfe) $236 \mathrm{cc}$ erhalten werden sollten. Auch die Kosten des anf die vorgeschlagene Weise gewonnenen Gases sind nur gering, sofern man die bei seiner Darstellung erhaltenen Rückstände nicht wegwirft, sondern behufs Aufarbeitung zurückstellt. Man muss sich hierbei erinnern, dass der Materialwerth des Sauerstoff entbindenden Gemisches nahezu erhalten bleibt, indem dieses mehr der 'Träger des Gases ist und nach seiner Ausnützung von Neuem imprägnirt werden kann. Dass sich dies ohne erhebliche Kosten bewerkstelligen lässt, ist durch die geringe Löslichkeit des Reactionsproductes (des Ferrocyankaliumbaryums) wesentlich mit bedingt, welche wir oben als einen sebr wichtigen Umstand hervcrgehoben haben. Man kann also den Materialwerth des Ferricyankaliums ganz eliminiren und annehmen, dass die Kosten der Aufarbeitung für $100 \mathrm{k}$ des Salzes etwa 30 Mark betragen werden, während der Werth des verbrauchten, etwa 80 proc. Baryumsuperoxyds mit 140 Mark für $100 \mathrm{k}$ in Rechnung zu ziehen ist.

Da nun nach der Gleichung:

$$
6,58: 0,16=100: x
$$

von $100 \mathrm{~g}$ Ferricyankalium immer $2,416 \mathrm{~g}$ Sauerstoff entwickelt werden, so liefern $100 \mathrm{k}$ des Salzes $2416 \mathrm{~g}$ oder $1689 \mathrm{l}$ Sauerstoff von $0^{\circ}$ und $760 \mathrm{~mm}$. Auf $100 \mathrm{k}$ Ferricyankalium kommen nach dem Ansatz

$$
6,58: 1,69=100: x
$$

rund $25,6 \mathrm{k} 100$ proc. oder $32 \mathrm{k} 80$ proc. Baryumsuperoxyd. Diese Menge liefert ebenfalls 1689 l Sauerstoff. Die Kosten dieser $2 \times 1689=3378 l$ betragen demnach aus den

$100 \mathrm{k}$ Ferricyankalium $\quad=30 \mathrm{M}$ aus den 32 k 80 proc. Baryumsuperoxyd $=44,80 \mathrm{M}$.

$7 \overline{4}, \overline{80 M}$.

Mitbin kommt nach dieser Berechnung das Iiter Sauerstoff auf 2,2 Pfennige zustehen. Wie man sieht, ist bei dieser Zusammenstellung der Werth des in den Rückständen enthaltenen und wieder zu gewinnenden Baryts gar nicht mit berücksichtigt, wodurch sich das Ergebniss noch gūnstiger stellen würde. Es sei hiermit darauf hingewiesen, dass die chemische Fabrik von H. Trommsdorff in Erfurt den Verkauf der Sauerstoff liefernden Mischung von Baryumsuperoxyd und Ferricyankalium übernommen hat und ebenso bereit ist, die Rückstände, welche bei der Darstellung von Sauerstoff nach meiner Methode gewonnen werden, wieder zurückzukaufen.
Für den Fall, dass man nicht an eine Aufbewahrung und Wiederverkauf des so leicht zu samnelnden Nebenproductes denkt, stellt sich das Sauerstoffgas wesentlich theurer. Es dürfte dann derPreis für $1 / 8$ bis 10 Pfennige betragen.

Vergleicht man nun das eben beschriebene Verfahren hinsichtlich der Kosten mit einigen anderen, welche am meisten im Gebrauch sind, so unterliegt es keinem Zweifel, dass alle die Verfahren der Sauerstoffdarstellung. welche mit Wasserstoffsuperoxyd arbeiten, viel theurer sind.

Einzig und allein ist es die Darstellung des Gases aus chlorsaurem Kali, welche der Theorie nach eine viel vortheilhaftere Ausbeute liefert. Können doch aus $100 \mathrm{k}$ Kaliumchlorat zum Preise von etwa 140 Mark gegen $27406 l$ Sauerstoff dargestellt werden, so dass sich derPreis des Liters auf 0,6 Pfennige stellen würde. Es ist a ber nicht zu vergessen, dass man behufs Verwendung dieses Salzes dasselbe mit Braunstein mischen und die Mischung längere Zeit erhitzen muss und dass schliesslich die hierbei benutzten fiefässe aus Glas oder Metall sehr büufig zu ergänzen sind, so dass unter Eiurechuung dieser Melurausgaben auch hier das Liter Sauerstoff gegen 2 Pfennige kosten dürfte.

Zudem kommt, dase das aus chlorsaurem Kali oder aus sauren Flüssigkeiten bereitete Gas (z. B. das nach Volhard's Methode aus Chlorkalk, Salzsäure und Wasserstoffsuperoxyd dargestellte) stets mit anderen Gasen, wie Chicr, Kohlensäure verunreinigt ist, während das ron mir in einer kalten und alkalischen Fiüssigkeit gewonnene gradezu als chemisch rein betrachtet werden muss.

Erwägt man nun noch, dass die leichte Handhaiung des Verfahrens es auch dem an chemischen Arbeiten gänzlich Ungeiibten gestattet, rasch und sicher jede beliebige Menge Sauerstoff zu entwickeln, so wird man zu der Überzeugung kommen, dass die Bereitung dieses Gases jetzt eine der leichtesten und gefahrlosesten Operationen geworden ist. Es empfiehlt sich daher mein Verfahren, zumal für Demonstrationszwecke in Schulen und Laboratorien, in denen es alle Ansprüche auf Bequemlichkeit, Gefahrlosigkeit und Billigkeit erfüllen dürfte, letzteres dunn, wenn man die aus ihm erhaltenen Rückstände von Ferrocyansalz nicht wegwirft, sondern behufs gelegentlicher Umwandlung in Ferricyansalz aufhebt und an die Fabrik zurückverkauft. 